\title{
Child Abuse Arrest Technique with Victims using Imageprocessing
}

\author{
Bavanidevi $B$, iswarya $R$, ishwarya $S$, seetha $K$
}

\begin{abstract}
Dynamic:In this methodology, attempts to manage a generous extent of human facial lead by seeing facial muscle exercises that produce expressions. Virtually most of the present vision systems for facial muscle movement area deal just with frontal-see face pictures and various wellsprings of photographic evidence are normally used by police forces to recognize suspects and setbacks of both on the web and disengaged crimes.Human properties, for instance, age, stature, weight, sex, hair shading, etc., are as often as possible used by cops and eyewitnesses in their depiction of unidentified suspects.In certain conditions, the age of the shocking loss can result in the affirmation of the bad behavior's grouping, e.g., tyke abuse investigations.Various motorized AI based techniques have been realized for the examination of automated pictures to distinguish fragile biometric attributes, for instance, age and sexual introduction, and hence help criminologists and masters in propelling their cases.
\end{abstract} vision)

\section{INTRODUCTION}

This paper tends to the PC supported identification of tyke sexual maltreatment (CSA) pictures, a test of developing significance in sight and sound crime scene investigation and security.In differentiation to past arrangements dependent on hash aggregates, record names, or the recovery of outwardly comparable pictures, we present a framework which utilizes visual acknowledgment systems to consequently recognize suspect materialWe build up a usage of the proposed calculation utilizing face recognition. Face discovery is chosen in light of the fact that a human face is regularly a district of intrigue. what's more, the nature of a face locale has huge effect on by and large picture quality. Likewise, face discovery is an all around contemplated zone in article location, and we approach various great face recognition calculations.

\section{RELATED WORKS}

ShubhangiP.Mankar et al proposed SMS based arrangement utilizing GPS framework to help guardians to follow their kids area continuously. These days, most cell phones are outfitted with area administrations capacities enabling us to get the gadget's geographic position continuously. The GPS and GSM based frameworks are utilized to follow the area of Child. It encourages the parent to get their tyke's area on an ongoing guide

Revised Manuscript Received on April 12, 2019.

Bavanidevi B,Bureau of Computer Science and Engineering, VSB Engineering College, Karur, Tamilnadu, Pin-639111 (bavab.tech@gmail.com)

iswarya $\mathbf{R}$,Bureau of Computer Science and Engineering, VSB Engineering College, Karur, Tamilnadu, Pin-639111

ishwarya S,Bureau of Computer Science and Engineering, VSB Engineering College, Karur, Tamilnadu, Pin-639111

seetha K,Bureau of Computer Science and Engineering, VSB Engineering College, Karur, Tamilnadu, Pin-639111
Keywords: $\quad$ Anaconda,spyder(software),opencv(computer

Poonam Patel et al proposed a framework is utilized for following the data of the lost tyke utilizing Google Map alongside the position and area of that tyke through GPS. This procedure work just by keeping the "following framework gadget" into the sack of that specific youngster, who is going to class or outside world and now on the off chance that on the off chance that that kid is lost or missed, at that point the guardians of that specific tyke can essentially follow him/her by communicating something specific name "TRACK" to the specific working gadget which has been kept inside that tyke pack. Thusly the guardians get the continuous area by accepting the accurate position of the youngster alongside the longitude and scope of that place then it will be replicated into the Google map and the area of that lost tyke can without much of a stretch be gotten to

\section{EXSTING SYSTEM}

To develop a dataset generator which outfits collections of a couple of unequal datasets and

structures a reasonable, curated dataset of cutting edge pictures remarked on with their looking at age and genderThis is completed in Evaluating Computerized Facial Age Estimation Techniques for Digital Forensics Furthermore, inventive AI systems and PC vision can reinforce electronic legitimate masters to finish motorized record request, standard unmistakable sorts of substance huge to a case in court, what's more, lessen the introduction of adolescent abuse material in cutting edge legitimate research focuses.

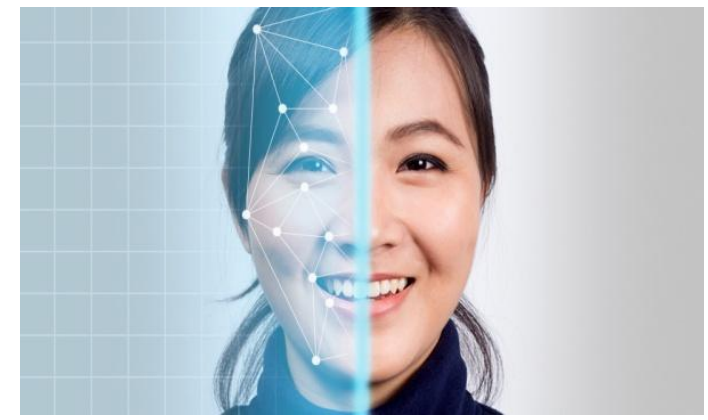

\section{PROPOSED SYSTEM\& RESULTS}

While human abilities to distinguish and recognize numerous features, for example, age, sex, ethnicity and outward appearances, can be cultivated by a speedy look at a computerized picture, machines are required to be prepared seriously so as to comprehend attributes present in photos. 


\section{CHILD ABUSE ARREST TECHNIQUE WITH VICTIMS USING IMAGEPROCESSING}

Checking the outside of a circle for information with probative esteem has for quite some time been a tedious assignment for scientific examiners. Hence our undertaking increment speed of procedure in measurable investigation.It additionally give security to reasonable document, for example, tyke misuse

\section{MODULE EXPLANATION}

Anaconda:Boa constrictor It is a free and open-source distributionofthe

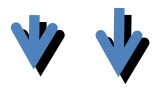
Python and $\mathrm{R}$ programming vernaculars for coherent computing(data science, AI applications, generous scale data taking care of, perceptive examination, etc.), that plans to streamline pack the officials and association. Pack variations are managed by the pack the officials system conda.

Boa constrictor Navigator is a work zone graphical UI (GUI) consolidated into Boa constrictor movement that empowers customers to dispatch applications and supervise conda packs, circumstances and channels without using heading line bearings. Pilot can check for groups on Anaconda Cloud or in a neighborhood Anaconda Repository, present them in a space, run the groups furthermore, revive them. It is open for Windows, macOS and Linux.

Open CV - Computer vision is a field software engineering that tackles engaging PCs to see, perceive and process pictures comparatively that human vision does, and after that give appropriate yield. It takes after presenting human knowledge and driving forces to a PC. When in doubt be that as it may, it is a troublesome endeavor to enable PCs to see pictures of different things. PC vision is solidly associated with man-made thinking, as the PC must decipher what it sees, and after that perform legitimate examination or act fittingly

Spyder-It is an open source cross-arrange facilitated improvement condition (IDE) for coherent programming in the Python language. It joins with different observable packages in the intelligent Python stack including NumPy, SciPy, pandas, IPython, and Cython, similarly as other open source programming. It is released under the MIT license

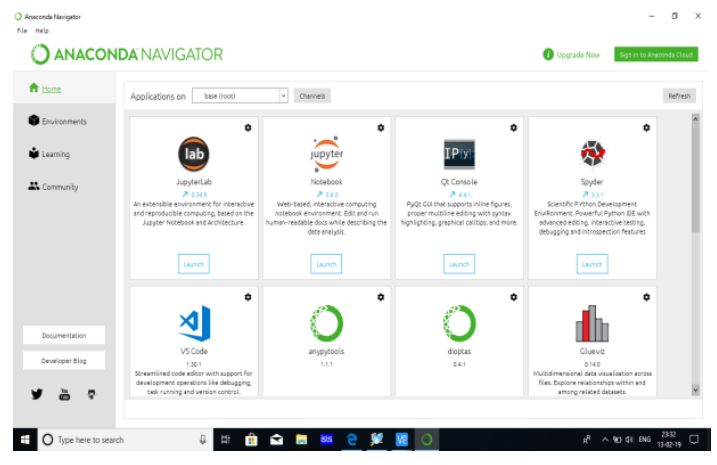

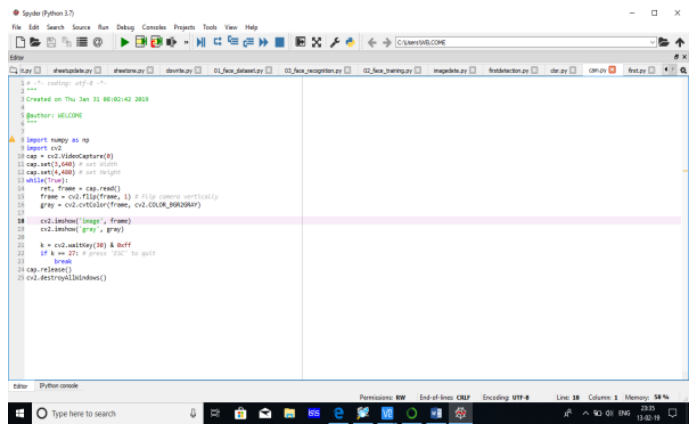

\section{REFERENCE}

1. H. Weber, A. Cruz Rodrigues, and A. Mateus, "Feeling and Mood in Design Thinking," Design Doctoral Conference'16: TRANSversality - Proceedings of the DDC third Conference, no. July, pp. 65-72, 2016.

2. G. Guo, "Human age estimation and sex characterization," Studies in Computational Intelligence, vol. 409, pp. 101-131, 2012.

3. R. Hurley, S. Prusty, H. Soroush, R. J. Dividers, J. Albrecht, E. Cecchet, B. N. Levine, M. Liberatore, B. Lynn, and J. Wolak, "Estimation and investigation of kid sex entertainment dealing on p2p systems," in Proceedings of the 22nd worldwide gathering on World Wide Web. ACM, 2013, pp. 631- 642.

4. J. Farina, M. Scanlon, N.- A. Le-Khac, and M.- T. Kechadi, "Outline of the Forensic Investigation of Cloud Services," in tenth International Conference on Availability, Reliability and Security (ARES 2015). Toulouse, France: IEEE, 08 2015, pp. 556- 565.

5. M. Aiken, M. Moran, and M. J. Berry, "Kid misuse material and the web: Cyberpsychology of online tyke related sex irritating," in 29th gathering of the INTERPOL Specialist Group on Crimes against Children, Lyons, France, September, 2011, pp. 5-7.

6. B. Jones, S. Pleno, and M. Wilkinson, "The utilization of irregular testing in examinations including tyke misuse material," Digital Investigation, vol. 9, pp. S99- S107, 2012.

7. T. Krone, "A typology of online kid erotic entertainment irritating," Trends and issues in wrongdoing and criminal equity, no. 279, pp. 1- 6, 2004.

8. Z. Erkin, M. Franz, J. Guajardo, S. Katzenbeisser, I. Lagendijk, and T. Toft, "Security saving face acknowledgment," in Proceedings of the ninth International Symposium on Privacy Enhancing Technologies, ser. PETS '09. Berlin, Heidelberg: Springer-Verlag, 2009, pp. 235- 253.

9. R. Rothe, R. Timofte, and L. V. Gool, "Profound desire for genuine and obvious age from a solitary picture without facial tourist spots," International Journal of Computer Vision (IJCV), July 2016.

10. P. J. Phillips, H. Wechsler, J. Huang, and P. J. Rauss, "The feret database and assessment strategy for faceacknowledgment calculations," Image and vision processing, vol. 16, no. 5, pp. 295-306, 1998.

11. A. P. Establishes, N. Orlans, W. Genevieve, and C. I. Watson, "Nist uncommon databse 32-numerous experience dataset ii (drugs ii)," NIST Interagency/Internal Report (NISTIR)7807, 2011 